\title{
Microwave assisted rapid synthesis of 4-amino-3, 4- dihydroquinolin-2-ones from azetidin-2-ones
}

\author{
Nilesh M. Shirode, Kiran C. Kulkarni, Vikas K. Gumaste, and \\ Abdul Rakeeb A. S. Deshmukh* \\ Division of Organic Chemistry (Synthesis), National Chemical Laboratory, \\ Pune - 411 008, India \\ E-mail: arasd@dalton.ncl.res.in
}

(received 16 Sept 04; accepted 05 Nov 04; published on the web 12 Nov 04)

\begin{abstract}
An efficient one-pot synthesis of 4-amino-3, 4-dihydroquinolin-2-ones from 3-(2-nitrophenyl)-1, 4-disubstituted azetidin-2-ones is described. Microwave assisted transfer hydrogenation of a nitro group followed by in situ $\beta$-lactam ring opening by the newly formed amino group is the key step in this synthesis.
\end{abstract}

Keywords: Azetidin-2-ones, 4-amino-3,4-dihydroquinolin-2-ones, transfer hydrogenation, microwave, ketenes, imines

\section{Introduction}

Apart from the substructure of widely used antibiotics ${ }^{1-3}$ such as penicillin, cephalosporins, monobactams etc., the azetidin-2-one ( $\beta$-lactam) skeleton has been recognized as a useful building block in stereoselective syntheses of biologically important compounds. ${ }^{4}$ This is mainly because there are many methods available to prepare them in large quantities. The strain energy associated with the four membered azetidin-2-one ring makes it susceptible for nucleophilic ring cleavage. This factor is also responsible for their application as synthon for various stereo selective syntheses of heterocyclic non $\beta$-lactam structures. ${ }^{5}$ Also, some of the synthetic $\beta$ lactams display interesting biological activities such as inhibition of prostate specific antigens, ${ }^{6}$ thrombin, ${ }^{7}$ human cytomegalovirus protein, ${ }^{8}$ cholesterol absorption, ${ }^{9}$ human leukocyte elastase ${ }^{10}$ and cysteine protease. ${ }^{11}$ As a consequence, the interest of organic chemists in the synthesis of new $\beta$-lactam derivatives remains high. Although, there are numerous methods available for the construction of the $\beta$-lactam ring, a widely used method is the [2+2] cyclocondensation of ketenes to imines, a process known as the Staudinger reaction. ${ }^{12-13}$ 


\section{Results and Discussion}

In continuation of our efforts towards the synthesis of substituted $\beta$-lactams via the Staudinger reaction $^{14}$ and their utility as synthons ${ }^{15}$ for the synthesis of various biologically important compounds, we herein report a rapid and practical synthesis of 4-amino-3, 4-dihydroquinolin-2ones from 1,3-disubstituted-4-(2-nitrophenyl)azetidin-2-ones via reduction of the nitro group followed by the intramolecular nucleophilic opening of the azetidin-2-one ring.

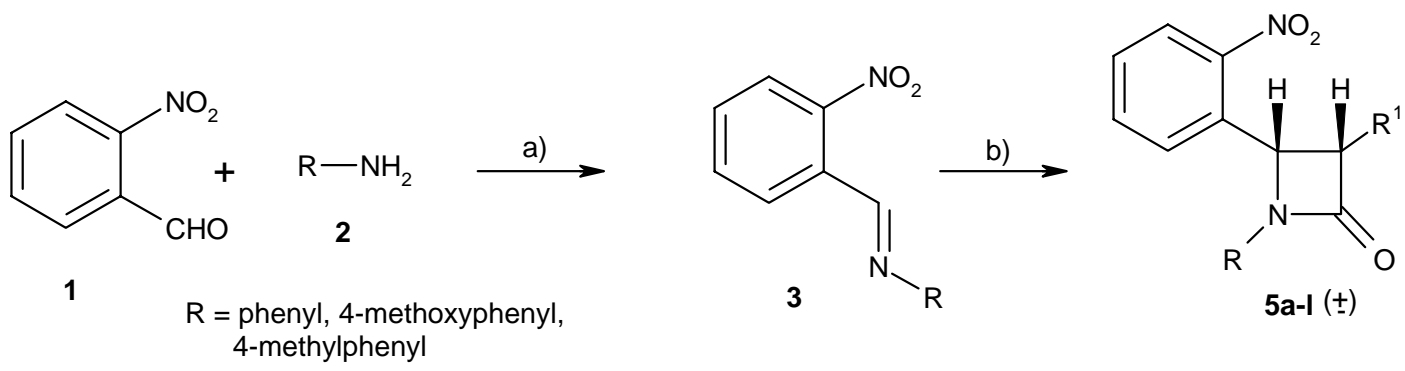

Reagents and conditions: a) $\mathrm{CH}_{2} \mathrm{Cl}_{2}$, anhyd. $\mathrm{MgSO}_{4}$, rt, $15 \mathrm{~h} \mathrm{~b}$ ) $\mathrm{R}_{1} \mathrm{CH}_{2} \mathrm{COCl}$ [4], $\mathrm{Et}_{3} \mathrm{~N}, \mathrm{CH}_{2} \mathrm{Cl}_{2}, 0{ }^{\circ} \mathrm{C}$ to rt, $18 \mathrm{~h}$

\section{Scheme 1}

Although, a solid supported synthesis of 4-amino-3, 4-dihydroquinolin-2-ones is reported, ${ }^{16}$ it is difficult to apply it for a gram-scale preparation. We have developed a practical synthesis of dihydroquinolinones, which can be adapted for a gram-scale preparation. Microwave assisted transfer hydrogenation of the nitro group followed by the nucleophilic opening of the $\beta$-lactam ring by the newly generated amino group is the key step in this synthesis.

Monocyclic 1,3-disubstituted-4-(2-nitrophenyl) azetidin-2-ones (5a-l) were prepared by [2+2] cycloaddition (Staudinger reaction) reaction of ketenes, generated in situ from substituted acetyl chlorides using tertiary amines and imines derived from reaction of 2-nitrobenzaldehyde with various amines. The cycloaddition reaction was highly stereoselective and gave cis $\beta$ lactams (5a-l) ( $J=5-6 \mathrm{~Hz}$ for cis $\beta$-lactam ring protons) in good to moderate yields (Table 1).

Initially the reduction of 5a was carried out by transfer hydrogenation using ammonium formate and $\mathrm{Pd} / \mathrm{C}(10 \%)$ in dry methanol at room temperature for $24 \mathrm{~h}$. Formation of amino- $\beta$ lactam 6 was observed in good yield along with trace amounts of cyclized 4-amino-3,4dihydroquinolin-2-one (7a). The cyclized product 7a was difficult to separate from amino $\beta$ lactam 6. However, its formation was deduced from IR and ${ }^{1} \mathrm{H}$ NMR spectra of the crude reaction mixture. The IR spectrum of amino- $\beta$-lactam 6 showed a characteristic $\beta$-lactam carbonyl absorption at $1730 \mathrm{~cm}^{-1}$ and amino group absorptions at $3398,3485 \mathrm{~cm}^{-1}$, while the cyclized product 7a showed the $\delta$-lactam carbonyl absorption at $1687 \mathrm{~cm}^{-1}$ and the NH absorption at $3373 \mathrm{~cm}^{-1}$. ${ }^{1} \mathrm{H}$ NMR spectrum of the amino- $\beta$-lactam 6 showed two doublets at $\delta$ 5.46 and 5.61 for the $\mathrm{C} 3$ and $\mathrm{C} 4$ cis- $\beta$-lactam ring protons $(J=4.7 \mathrm{~Hz}$ for the cis-isomer $)$. 
Table 1. Synthesis of 4-(2-nitrophenyl)-1,3-disubstituted azetidin-2-ones (5a-l)

\begin{tabular}{llllll}
\hline Entry No & Compound & $\mathrm{R}$ & $\mathrm{R}^{1}$ & Yield $^{\mathrm{a}}(\%)$ & $\mathrm{Mp}\left({ }^{\circ} \mathrm{C}\right)$ \\
\hline 1 & $\mathbf{5 a}$ & 4-Methoxyphenyl & Phenoxy & 90 & 142 \\
2 & $\mathbf{5 b}$ & 4-Methoxyphenyl & Methoxy & 65 & 158 \\
3 & $\mathbf{5 c}$ & 4-Methoxyphenyl & Benzyloxy & 82 & 181 \\
4 & $\mathbf{5 d}$ & 4-Methoxyphenyl & Acetoxy & 79 & 180 \\
5 & $\mathbf{5 e}$ & Phenyl & Phenoxy & 74 & 137 \\
6 & $\mathbf{5 f}$ & Phenyl & Methoxy & 68 & 135 \\
7 & $\mathbf{g}$ & Phenyl & Benzyloxy & 70 & 143 \\
8 & $5 \mathbf{h}$ & Phenyl & Acetoxy & 65 & 180 \\
9 & $5 \mathbf{i}$ & $p$-Tolyl & Phenoxy & 82 & 153 \\
10 & $\mathbf{5 j}$ & $p$-Tolyl & Methoxy & 63 & 157 \\
11 & $\mathbf{5 k}$ & $p$-Tolyl & Benzyloxy & 79 & 165 \\
12 & $\mathbf{5 l}$ & $p$-Tolyl & Acetoxy & 72 & 157 \\
\hline
\end{tabular}

${ }^{\text {a }}$ Isolated yields.

The amino $\beta$-lactam underwent smooth cyclization by refluxing in methanol for $2 \mathrm{~h}$ to give the dihydroquinolin-2-one 7a in quantitative yield. The ${ }^{1} \mathrm{H}$ NMR spectrum of 7a showed two doublets at $\delta 5.13$ and 5.23 for $\mathrm{C} 3$ and $\mathrm{C} 4$ trans- $\delta$-lactam ring protons $(J=8.6 \mathrm{~Hz})$. A direct transfer hydrogenation of 5a was also tried in refluxing methanol for $2 \mathrm{~h}$, which gave mixtures of amino- $\beta$-lactam 6 and 4-amino-3,4-dihydroqunolin-2-one 7a along with several other unidentified products.
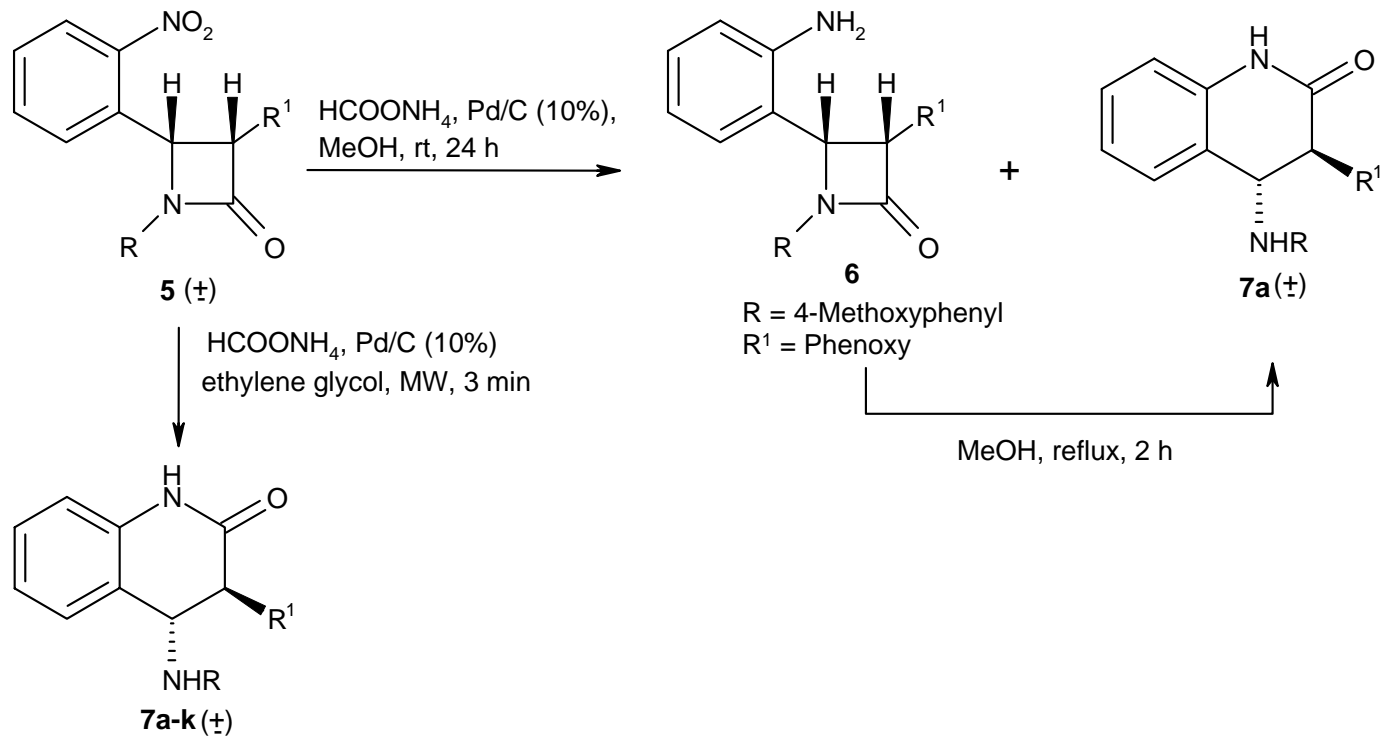

\section{Scheme 2}


We envisaged that transfer hydrogenation of 5a under microwave irradiation (MW) would directly give us dihydroquinolin-2-one 7a. The use of microwave irradiation in enhancing chemical transformation has gained considerable attention in recent years due to several advantages such as a high reaction rate and pure product formation with higher yields. ${ }^{17}$ Although the reason for the rate enhancement is not clear, selective absorption of microwave energy by polar molecules or transition states may be responsible for the acceleration of the reaction.

Microwave irradiation of $\mathbf{5 a}$ in the presence of ammonium formate and catalytic amounts of $\mathrm{Pd} / \mathrm{C}(10 \%)$ in the presence of a small quantity of ethylene glycol was carried out in an open glass vessel using a domestic microwave. The reaction was over in just three minutes at $60 \%$ power of the microwave oven. The reaction mixture was diluted with water, methylene chloride and the catalyst was removed by filtration through a small bed of celite. The filtrate was extracted with methylene chloride and the removal of methylene chloride under reduced pressure gave almost pure product 7a in very good yield, which was further purified by crystallization from ethyl acetate-petroleum ether mixture.

This product was formed by the reduction of the nitro group followed by the nucleophilic $\beta$ lactam ring cleavage with the newly generated amino group. Several 4-amino-3,4dihydroquinolin-2-ones (7a-k) were prepared by transfer hydrogenation under microwave irradiation in very good yields (Table 2 ). In case of acetoxy compounds $\mathbf{7 g}, \mathbf{k}$ (Table 2 , entries 7 and 11) a small amount of the corresponding uncyclized amino- $\beta$-lactam was also observed along with the required dihydroquinolin-2-one, which was removed by crystallization from an ethyl acetate-pet-ether mixture.

Table 2. Synthesis of 4-amino-3, 4-dihydroquinolin-2-ones (7a-k)

\begin{tabular}{llllll}
\hline Entry No & Compound & $\mathrm{R}$ & $\mathrm{R}^{1}$ & Yield $^{\mathrm{a}}(\%)$ & $\mathrm{Mp}\left({ }^{\circ} \mathrm{C}\right)$ \\
\hline 1 & $\mathbf{7 a}$ & 4-Methoxyphenyl & Phenoxy & 89 & 229 \\
2 & $\mathbf{7 b}$ & 4-Methoxyphenyl & Methoxy & 90 & 208 \\
3 & $\mathbf{7 c}$ & 4-Methoxyphenyl & Benzyloxy & 86 & 201 \\
4 & $\mathbf{7 d}$ & Phenyl & Phenoxy & 87 & 225 \\
5 & $\mathbf{7 e}$ & Phenyl & Methoxy & 81 & 209 \\
6 & $\mathbf{7 f}$ & Phenyl & Benzyloxy & 82 & 218 \\
7 & $\mathbf{7 g}$ & Phenyl & Acetoxy & 74 & 221 \\
8 & $\mathbf{7 h}$ & $p$-Tolyl & Phenoxy & 87 & 248 \\
9 & $\mathbf{7 i}$ & $p$-Tolyl & Methoxy & 86 & 211 \\
10 & $\mathbf{7 j}$ & $p$-Tolyl & Benzyloxy & 78 & 203 \\
11 & $\mathbf{7 k}$ & $p$-Tolyl & Acetoxy & 77 & 238 \\
\hline
\end{tabular}

${ }^{\text {a }}$ Isolated yields. 
In conclusion, we have demonstrated a simple and efficient one-pot preparation of 4-amino3, 4-dihydroquinolin-2-ones from 3-(2-nitrophenyl)-1,4-disubstituted azetidin-2-ones. Microwave assisted transfer hydrogenation of the nitro group followed by the in situ $\beta$-lactam ring opening by the newly formed amino group is the key step in this synthesis.

\section{Experimental Section}

General Procedures. ${ }^{1} \mathrm{H}$ NMR and ${ }^{13} \mathrm{C}$ NMR Spectra were recorded in a $\mathrm{CDCl}_{3}$ solution on a Bruker AC 200, Bruker MSL-300 and Bruker DRX-500 spectrometer and chemical shifts are reported in ppm downfield from TMS for ${ }^{1} \mathrm{H}$ NMR. Infrared spectra were recorded on Shimadzu FT IR-8400 using sodium chloride optics. Melting points were determined on a Thermonik Campbell melting point apparatus and were uncorrected. The microanalyses were performed on a Carlo-Erba, CHNS-O EA 1108 elemental analyzer. Microwave irradiation was carried out in an open glass vessel using a domestic microwave oven (800 Watt, BPL-make).

\section{General procedure for the synthesis of azetidine-2-ones (5a-l)}

To a solution of an imine $3(5 \mathrm{mmol})$ and triethylamine $(20 \mathrm{mmol})$ in dry methylene chloride $(20 \mathrm{~mL})$ was added dropwise a solution of an acid chloride $4(7.5 \mathrm{mmol})$ in dry methylene chloride $(10 \mathrm{~mL})$ with stirring at $0{ }^{\circ} \mathrm{C}$ in about $20 \mathrm{~min}$. The reaction mixture was then allowed to warm up to room temperature and stirred for $14 \mathrm{~h}$. It was then washed with water $(10 \mathrm{~mL})$, saturated sodium bicarbonate $(10 \mathrm{~mL})$, brine $(10 \mathrm{~mL})$ and dried over anhyd. $\mathrm{Na}_{2} \mathrm{SO}_{4}$. The solvent was removed in vacuo to give crude azetidine-2-one, which was recrystallized from methanol to get pure azetidine-2-one.

1-(4-Methoxyphenyl)-4-(2-nitrophenyl)-3-phenoxyazetidin-2-one (5a). Yield, 1.76 g, 90\%; yellow crystalline solid, mp $142{ }^{\circ} \mathrm{C}$; IR $\left(\mathrm{CHCl}_{3}\right) 1757 \mathrm{~cm}^{-1} ;{ }^{1} \mathrm{H} \mathrm{NMR}\left(\mathrm{CDCl}_{3}, 200 \mathrm{MHz}\right) \delta 8.26-$ $8.21(\mathrm{~m}, 1 \mathrm{H}), 7.64-7.49(\mathrm{~m}, 3 \mathrm{H}), 7.36(\mathrm{~d}, J=9.4 \mathrm{~Hz}, 2 \mathrm{H}), 7.26-7.17(\mathrm{~m}, 2 \mathrm{H}), 7.00-6.86(\mathrm{~m}, 5 \mathrm{H})$, $6.16(\mathrm{~d}, J=5.1 \mathrm{~Hz}, 1 \mathrm{H}), 5.71(\mathrm{~d}, J=5.5 \mathrm{~Hz}, 1 \mathrm{H}), 3.79(\mathrm{~s}, 3 \mathrm{H}) ;{ }^{13} \mathrm{C} \mathrm{NMR}\left(\mathrm{CDCl}_{3}, 125.76 \mathrm{MHz}\right)$ $\delta 163.07,157.12,156.64,148.02,133.86,130.28,130.13,129.32,129.14,129.06,125.28$, 122.58, 118.47, 116.24, 114.50, 82.11, 58.97, 55.34. Anal. Calcd. for $\mathrm{C}_{22} \mathrm{H}_{18} \mathrm{~N}_{2} \mathrm{O}_{5}$ : C, 67.69; $\mathrm{H}$, 4.61; N, 7.18. Found: C, 67.93; H, 4.48; N, 7.51.

3-Methoxy-1-(4-methoxyphenyl)-4-(2-nitrophenyl) azetidin-2-one (5b). Yield, $1.07 \mathrm{~g}, 65 \%$; white solid, mp $158{ }^{\circ} \mathrm{C}$; IR $\left(\mathrm{CHCl}_{3}\right) 1753 \mathrm{~cm}^{-1} ;{ }^{1} \mathrm{H} \mathrm{NMR}\left(\mathrm{CDCl}_{3}, 500 \mathrm{MHz}\right) \delta 8.23-8.21(\mathrm{~m}, 1 \mathrm{H})$, 7.61-7.58 (m, 1H), 7.53-7.50 (m, 1H), $7.45(\mathrm{~d}, J=7.8 \mathrm{~Hz}, 1 \mathrm{H}), 7.28(\mathrm{~d}, J=9.2 \mathrm{~Hz}, 2 \mathrm{H}), 6.84(\mathrm{~d}$, $J=9.2 \mathrm{~Hz}, 2 \mathrm{H}), 5.88(\mathrm{~d}, J=5.0 \mathrm{~Hz}, 1 \mathrm{H}), 5.00(\mathrm{~d}, J=5.0 \mathrm{~Hz}, 1 \mathrm{H}), 3.77(\mathrm{~s}, 3 \mathrm{H}), 3.37(\mathrm{~s}, 3 \mathrm{H}) ;{ }^{13} \mathrm{C}$ NMR $\left(\mathrm{CDCl}_{3}, 125.76 \mathrm{MHz}\right) \delta 164.35,156.73,148.34,133.82,130.74,130.54,129.38,128.94$, 125.34, 118.53, 114.65, 85.65, 59.55, 59.49, 55.49. Anal. Calcd. for $\mathrm{C}_{17} \mathrm{H}_{16} \mathrm{~N}_{2} \mathrm{O}_{5}$ : C, 62.19; $\mathrm{H}$, 4.87, N, 8.53. Found: C, 62.37; H, 4.64; N, 8.49.

3-Benzyloxy-1-(4-methoxyphenyl)-4-(2-nitrophenyl) azetidin-2-one (5c). Yield, $1.66 \mathrm{~g}, 82 \%$; white solid, $\mathrm{mp} 181^{\circ} \mathrm{C}$; IR $\left(\mathrm{CHCl}_{3}\right) 1753 \mathrm{~cm}^{-1} ;{ }^{1} \mathrm{H} \mathrm{NMR}\left(\mathrm{CDCl}_{3}, 200 \mathrm{MHz}\right) \delta 8.25-8.20(\mathrm{~m}, 1 \mathrm{H})$, 
7.64-7.49 (m, 3H), 7.33-7.23 (m, 5H), 7.08-7.03 (m, 2H), $6.86(\mathrm{~d}, J=9.0 \mathrm{~Hz}, 2 \mathrm{H}), 5.91(\mathrm{~d}, J=$ $5.1 \mathrm{~Hz}, 1 \mathrm{H}), 5.22(\mathrm{~d}, J=5.1 \mathrm{~Hz}, 1 \mathrm{H}), 4.63(\mathrm{~d}, J=11.8 \mathrm{~Hz}, 1 \mathrm{H}), 4.54(\mathrm{~d}, J=11.7 \mathrm{~Hz}, 1 \mathrm{H}), 3.78$ $(\mathrm{s}, 3 \mathrm{H}) ;{ }^{13} \mathrm{C} \mathrm{NMR}\left(\mathrm{CDCl}_{3}, 125.76 \mathrm{MHz}\right) \delta 164.20,156.54,148.20,136.54,133.84,130.73$, 130.38, 129.41, 128.91, 128.21,127.78, 127.51, 125.29, 118.42, 114.49, 83.41, 73.18, 59.49, 55.39. Anal. Calcd. for $\mathrm{C}_{23} \mathrm{H}_{20} \mathrm{~N}_{2} \mathrm{O}_{5}$ : C, 68.30; H, 4.99; N, 6.93. Found: C, 68.14; H, 5.10; N, 6.71 .

3-Acetoxy-1-(4-methoxyphenyl)-4-(2-nitrophenyl) azetidin-2-one (5d). Yield, $1.41 \mathrm{~g}, 79 \%$; creamy white solid, mp $180{ }^{\circ} \mathrm{C}$; IR $\left(\mathrm{CHCl}_{3}\right) 1759 \mathrm{~cm}^{-1} ;{ }^{1} \mathrm{H} \mathrm{NMR}\left(\mathrm{CDCl}_{3}, 200 \mathrm{MHz}\right) \delta 8.23-8.18$ $(\mathrm{m}, 1 \mathrm{H}), 7.65-7.48(\mathrm{~m}, 3 \mathrm{H}), 7.31(\mathrm{~d}, J=9.0 \mathrm{~Hz}, 2 \mathrm{H}), 6.87(\mathrm{~d}, J=9.0 \mathrm{~Hz}, 2 \mathrm{H}), 6.36(\mathrm{~d}, J=5.5$ $\mathrm{Hz}, 1 \mathrm{H}), 6.05(\mathrm{~d}, J=5.5 \mathrm{~Hz}, 1 \mathrm{H}), 3.78(\mathrm{~s}, 3 \mathrm{H}), 1.77(\mathrm{~s}, 3 \mathrm{H}) ;{ }^{13} \mathrm{C} \mathrm{NMR}\left(\mathrm{CDCl}_{3}, 125.76 \mathrm{MHz}\right) \delta$ $168.18,161.62,156.69,148.08,133.75,129.95,129.37,129.23,129.08,125.29,118.44,114.49$, 75.76, 58.23, 55.34, 19.78. Anal. Calcd. for $\mathrm{C}_{18} \mathrm{H}_{16} \mathrm{~N}_{2} \mathrm{O}_{6}$ : C, 60.66; H, 4.53; N, 7.86. Found: C, $60.91 ; \mathrm{H}, 4.35 ; \mathrm{N}, 7.86$.

4-(2-Nitrophenyl)-3-phenoxy-1-phenyl azetidin-2-one (5e). Yield, $1.33 \mathrm{~g}$, 74\%; creamy white solid, mp $137{ }^{\circ} \mathrm{C}$; IR $\left(\mathrm{CHCl}_{3}\right) 1759 \mathrm{~cm}^{-1} ;{ }^{1} \mathrm{H} \mathrm{NMR}\left(\mathrm{CDCl}_{3}, 200 \mathrm{MHz}\right) \delta 8.29-8.24(\mathrm{~m}, 1 \mathrm{H}), 7.68-$ $7.51(\mathrm{~m}, 3 \mathrm{H}), 7.47-7.32(\mathrm{~m}, 4 \mathrm{H}), 7.28-7.25(\mathrm{~m}, 1 \mathrm{H}), 7.21(\mathrm{~d}, J=8.3 \mathrm{~Hz}, 2 \mathrm{H}), 7.00(\mathrm{~d}, J=7.5 \mathrm{~Hz}$, $1 \mathrm{H}), 6.91(\mathrm{~d}, J=7.8 \mathrm{~Hz}, 2 \mathrm{H}), 6.22(\mathrm{~d}, J=5.5 \mathrm{~Hz}, 1 \mathrm{H}), 5.73(\mathrm{~d}, J=5.4 \mathrm{~Hz}, 1 \mathrm{H}) ;{ }^{13} \mathrm{C}$ NMR $\left(\mathrm{CDCl}_{3}, 75.48 \mathrm{MHz}\right) \delta 163.58,157.11,148.08,136.88,133.74,129.89,129.28,129.07,125.19$, 124.79, 122.60, 117.16, 116.28, 82.13, 58.90. Anal. Calcd. for $\mathrm{C}_{21} \mathrm{H}_{16} \mathrm{~N}_{2} \mathrm{O}_{4}: \mathrm{C}, 69.98 ; \mathrm{H}, 4.48 ; \mathrm{N}$, 7.77. Found: C, 69.90; H, 4.58; N, 7.65.

3-Methoxy-4-(2-nitrophenyl)-1-phenyl azetidin-2-one (5f). Yield, $1.02 \mathrm{~g}$, 68\%; pale yellow solid, mp $135{ }^{\circ} \mathrm{C}$; IR $\left(\mathrm{CHCl}_{3}\right) 1757 \mathrm{~cm}^{-1} ;{ }^{1} \mathrm{H} \mathrm{NMR}\left(\mathrm{CDCl}_{3}, 200 \mathrm{MHz}\right) \delta 8.27-8.22(\mathrm{~m}, 1 \mathrm{H}), 7.61$ $7.48(\mathrm{~m}, 3 \mathrm{H}), 7.34-7.30(\mathrm{~m}, 4 \mathrm{H}), 7.27-7.13(\mathrm{~m}, 1 \mathrm{H}), 5.94(\mathrm{~d}, J=5.0 \mathrm{~Hz}, 1 \mathrm{H}), 5.03(\mathrm{~d}, J=5.5 \mathrm{~Hz}$, $1 \mathrm{H}), 3.38(\mathrm{~s}, 3 \mathrm{H}) ;{ }^{13} \mathrm{C} \mathrm{NMR}\left(\mathrm{CDCl}_{3}, 75.48 \mathrm{MHz}\right) \delta 164.77,148.14,136.94,133.89,130.41$, $129.19,128.82,125.19,124.58,117.38,117.10,85.42,59.30,58.11$. Anal. Calcd. for $\mathrm{C}_{16} \mathrm{H}_{14} \mathrm{~N}_{2} \mathrm{O}_{4}$ : C, 64.41; H, 4.74; N, 9.39. Found: C, 64.14; H, 4.72; N, 9.28.

3-Benzyloxy-4-(2-nitrophenyl)-1-phenyl azetidin-2-one (5g). Yield, $1.31 \mathrm{~g}, 70 \%$; creamy white solid, mp $143{ }^{\circ} \mathrm{C}$; IR $\left(\mathrm{CHCl}_{3}\right) 1755 \mathrm{~cm}^{-1} ;{ }^{1} \mathrm{H} \mathrm{NMR}\left(\mathrm{CDCl}_{3}, 200 \mathrm{MHz}\right) \delta 8.26-8.21(\mathrm{~m}, 1 \mathrm{H})$, 7.66-7.48 (m, 3H), 7.38-7.35 (m, 2H), $7.33(\mathrm{~d}, J=2.4 \mathrm{~Hz}, 2 \mathrm{H}), 7.28(\mathrm{~d}, J=1.2 \mathrm{~Hz}, 1 \mathrm{H}), 7.24(\mathrm{~d}$, $J=2.4 \mathrm{~Hz}, 2 \mathrm{H}), 7.18-7.12(\mathrm{~m}, 1 \mathrm{H}), 7.08(\mathrm{~d}, J=3.5 \mathrm{~Hz}, 1 \mathrm{H}), 7.04(\mathrm{~d}, J=2.3 \mathrm{~Hz}, 1 \mathrm{H}), 5.96(\mathrm{~d}, J$ $=5.1 \mathrm{~Hz}, 1 \mathrm{H}), 5.23(\mathrm{~d}, J=5.5 \mathrm{~Hz}, 1 \mathrm{H}), 4.65(\mathrm{~d}, J=11.7 \mathrm{~Hz}, 1 \mathrm{H}), 4.55(\mathrm{~d}, J=11.7 \mathrm{~Hz}, 1 \mathrm{H}) ;{ }^{13} \mathrm{C}$ NMR $\left(\mathrm{CDCl}_{3}, 75.48 \mathrm{MHz}\right) \delta 164.71,148.26,136.97,136.54,133.71,130.53,129.22,128.85$, $128.18,127.75,127.45,125.22,124.61,117.13,83.35,73.19,73.19,59.39$. Anal. Calcd. for $\mathrm{C}_{22} \mathrm{H}_{18} \mathrm{~N}_{2} \mathrm{O}_{4}$ : C, 70.57; H, 4.86; N, 7.48. Found: C, 70.42; H, 4.93; N, 7.42.

3-Acetoxy-4-(2-nitrophenyl)-1-phenyl azetidin-2-one (5h). Yield, 1.06 g, 65\%; creamy white solid, mp $180{ }^{\circ} \mathrm{C}$; IR $\left(\mathrm{CHCl}_{3}\right) 1755 \mathrm{~cm}^{-1}$; ${ }^{1} \mathrm{H} \mathrm{NMR}\left(\mathrm{CDCl}_{3}, 200 \mathrm{MHz}\right) \delta 8.23-8.18(\mathrm{~m}, 1 \mathrm{H}), 7.68-$ $7.47(\mathrm{~m}, 4 \mathrm{H}), 7.38-7.26(\mathrm{~m}, 3 \mathrm{H}), 7.19-7.10(\mathrm{~m}, 1 \mathrm{H}), 6.36(\mathrm{~d}, J=5.5 \mathrm{~Hz}, 1 \mathrm{H}), 6.09(\mathrm{~d}, J=5.5 \mathrm{~Hz}$, $1 \mathrm{H}), 1.77(\mathrm{~s}, 3 \mathrm{H}) ;{ }^{13} \mathrm{C} \mathrm{NMR}\left(\mathrm{CDCl}_{3}, 75.48 \mathrm{MHz}\right) \delta 167.92,162.09,148.02,136.48,133.61$, 129.28, 129.16, 128.91, 125.16, 124.76, 117.01, 75.63, 58.08, 19.53. Anal. Calcd. for $\mathrm{C}_{17} \mathrm{H}_{14} \mathrm{~N}_{2} \mathrm{O}_{5}$ : C, 62.57; H, 4.33; N, 8.59. Found: $\mathrm{C}, 62.50 ; \mathrm{H}, 4.28 ; \mathrm{N}, 8.42$. 
4-(2-Nitrophenyl)-3-phenoxy-1-p-tolyl azetidin-2-one (5i). Yield, $1.53 \mathrm{~g}, 82 \%$; pale yellow solid, mp $153{ }^{\circ} \mathrm{C}$; IR $\left(\mathrm{CHCl}_{3}\right) 1759 \mathrm{~cm}^{-1}$; ${ }^{1} \mathrm{H} \mathrm{NMR}\left(\mathrm{CDCl}_{3}, 200 \mathrm{MHz}\right) \delta 8.26-8.21(\mathrm{~m}, 1 \mathrm{H}), 7.64-$ $7.50(\mathrm{~m}, 3 \mathrm{H}), 7.32-7.13(\mathrm{~m}, 6 \mathrm{H}), 6.99-6.87(\mathrm{~m}, 3 \mathrm{H}), 6.17(\mathrm{~d}, J=5.5 \mathrm{~Hz}, 1 \mathrm{H}), 5.70(\mathrm{~d}, J=5.5$ $\mathrm{Hz}, 1 \mathrm{H}), 2.32(\mathrm{~s}, 3 \mathrm{H}) ;{ }^{13} \mathrm{C} \mathrm{NMR}\left(\mathrm{CDCl}_{3}, 75.48 \mathrm{MHz}\right) \delta 163.37,157.17,148.14,134.59,133.74$, 130.13 , 129.80, 129.31, 129.16, 129.01, 125.19, 122.60, 117.13, 116.31, 82.19, 58.90, 20.75; Anal. Calcd. for $\mathrm{C}_{22} \mathrm{H}_{18} \mathrm{~N}_{2} \mathrm{O}_{4}$ : C, 70.57; H, 4.85; N, 7.48. Found: C, 70.35; H, 4.96; N, 7.42.

3-Methoxy-4-(2-nitrophenyl)-1-p-tolyl azetidin-2-one (5j). Yield, $0.98 \mathrm{~g}$, 63\%; pale yellow solid, mp $157{ }^{\circ} \mathrm{C}$; IR $\left(\mathrm{CHCl}_{3}\right) 1753 \mathrm{~cm}^{-1} ;{ }^{1} \mathrm{H} \mathrm{NMR}\left(\mathrm{CDCl}_{3}, 200 \mathrm{MHz}\right) \delta 8.27-8.22(\mathrm{~m}, 1 \mathrm{H}), 7.64-$ $7.43(\mathrm{~m}, 3 \mathrm{H}), 7.26-7.10(\mathrm{~m}, 4 \mathrm{H}), 5.91(\mathrm{~d}, J=5.0 \mathrm{~Hz}, 1 \mathrm{H}), 5.02(\mathrm{~d}, J=5.5 \mathrm{~Hz}, 1 \mathrm{H}), 3.38(\mathrm{~s}, 3 \mathrm{H})$, $2.32(\mathrm{~s}, 3 \mathrm{H}) ;{ }^{13} \mathrm{C} \mathrm{NMR}\left(\mathrm{CDCl}_{3}, 75.48 \mathrm{MHz}\right) \delta 164.50,148.17,134.53,134.29,133.64,130.56$, 129.68, 129.19, 128.76, 125.16, 117.04, 85.42, 59.36, 20.72. Anal. Calcd. for $\mathrm{C}_{17} \mathrm{H}_{16} \mathrm{~N}_{2} \mathrm{O}_{4}$ : C, 65.37; H, 5.17; N, 8.97. Found: C, 65.44; H, 5.39; N, 9.03.

3-Benzyloxy-4-(2-nitrophenyl)-1-p-tolyl azetidin-2-one (5k). Yield, $1.53 \mathrm{~g}, 79 \%$; creamy white solid, mp $165^{\circ} \mathrm{C}$; IR $\left(\mathrm{CHCl}_{3}\right) 1755 \mathrm{~cm}^{-1} ;{ }^{1} \mathrm{H} \mathrm{NMR}\left(\mathrm{CDCl}_{3}, 200 \mathrm{MHz}\right) \delta 8.25-8.20(\mathrm{~m}, 1 \mathrm{H})$, 7.64-7.48 (m, 3H), 7.26-7.24 (m, 5H), 7.22-7.08 (m, 4H), $5.92(\mathrm{~d}, J=5.1 \mathrm{~Hz}, 1 \mathrm{H}), 5.21(\mathrm{~d}, J=$ $5.5 \mathrm{~Hz}, 1 \mathrm{H}), 4.64(\mathrm{~d}, J=12.2 \mathrm{~Hz}, 1 \mathrm{H}), 4.54(\mathrm{~d}, J=11.8 \mathrm{~Hz}, 1 \mathrm{H}), 2.31(\mathrm{~s}, 3 \mathrm{H}) ;{ }^{13} \mathrm{C}$ NMR $\left(\mathrm{CDCl}_{3}, 75.48 \mathrm{MHz}\right) \delta 164.44,148.20,136.54,134.50,134.29,133.67,130.65,129.68,129.31$, $128.76,128.12,127.69,127.42,125.16,117.04,83.35,73.12,59.33,20.72$. Anal. Calcd. for $\mathrm{C}_{23} \mathrm{H}_{20} \mathrm{~N}_{2} \mathrm{O}_{4}$ : C, 71.11; H, 5.20; N, 7.21. Found: C, 70.80; H, 5.27; N, 6.96.

3-Acetoxy-4-(2-nitrophenyl)-1-p-tolyl azetidin-2-one (5l). Yield, $1.23 \mathrm{~g}, 72 \%$; pale brown solid, mp $157{ }^{\circ} \mathrm{C}$; IR $\left(\mathrm{CHCl}_{3}\right) 1759 \mathrm{~cm}^{-1}$; ${ }^{1} \mathrm{H} \mathrm{NMR}\left(\mathrm{CDCl}_{3}, 200 \mathrm{MHz}\right) \delta 8.23-8.19(\mathrm{~m}, 1 \mathrm{H})$, 7.64$7.47(\mathrm{~m}, 3 \mathrm{H}), 7.26-7.11(\mathrm{~m}, 4 \mathrm{H}), 6.35(\mathrm{~d}, J=5.5 \mathrm{~Hz}, 1 \mathrm{H}), 6.05(\mathrm{~d}, J=5.5 \mathrm{~Hz}, 1 \mathrm{H}), 2.31(\mathrm{~s}, 3 \mathrm{H})$, $1.77(\mathrm{~s}, 3 \mathrm{H}) ;{ }^{13} \mathrm{C} \mathrm{NMR}\left(\mathrm{CDCl}_{3}, 75.48 \mathrm{MHz}\right) \delta 168.01,161.91,148.14,134.65,134.13,133.61$, $129.74,129.28,129.04,125.19,117.04,75.72,58.11,20.66,19.62$. Anal. Calcd. for $\mathrm{C}_{18} \mathrm{H}_{16} \mathrm{~N}_{2} \mathrm{O}_{5}$ : C, 63.52; H, 4.75; N, 8.23. Found: C, 63.71; H, 4.65; N, 7.90.

Transfer hydrogenation of 1-(4-methoxyphenyl)-4-(2-nitrophenyl)-3-phenoxyazetidin-2-one (5a). To a solution of 5 a $(0.195 \mathrm{~g}, 0.5 \mathrm{mmol})$ in dry methanol $(3 \mathrm{~mL})$ was added ammonium formate $(0.157 \mathrm{~g}, 2.5 \mathrm{mmol})$ followed by $\mathrm{Pd} / \mathrm{C}(10 \%, 30 \mathrm{mg})$. This reaction mixture was stirred at room temperature under argon for $24 \mathrm{~h}$, then filtered through a short celite bed, and washed with methylene chloride $(15 \mathrm{~mL})$. The filtrate was diluted with water $(2 \mathrm{~mL})$, the organic layer was separated and dried over anhyd. $\mathrm{Na}_{2} \mathrm{SO}_{4}$. The solvent was removed under vacuum to get 4(2-aminophenyl)-1-p-methoxyphenyl-3-phenoxy azetidin-2-one (6) as a pale yellow solid (0.176 g, 98\%), mp 195-199 ${ }^{\circ} \mathrm{C}$; IR $\left(\mathrm{CHCl}_{3}\right) 3485,3398,1730 \mathrm{~cm}^{-1} ;{ }^{1} \mathrm{H} \mathrm{NMR}\left(\mathrm{CDCl}_{3}, 200 \mathrm{MHz}\right)$ $\delta$ 7.41-6.76 (m, 13H), $5.61(\mathrm{~d}, J=4.7 \mathrm{~Hz}, 1 \mathrm{H}), 5.46(\mathrm{~d}, J=4.7 \mathrm{~Hz}, 1 \mathrm{H}), 3.77(\mathrm{~s}, 3 \mathrm{H}) ;{ }^{13} \mathrm{C}$ NMR $\left(\right.$ DMSO-d $\left._{6}, 125.76 \mathrm{MHz}\right) \delta 162.72,157.10,156.05,147.12,130.19,129.38,128.68,127.37$, $121.92,118.55,116.11,115.63,115.36,114.67,80.95,56.17,55.36$.

The above solid was dissolved in methanol $(10 \mathrm{~mL})$ and refluxed for $2 \mathrm{~h}$. The solvent was removed under reduced pressure to get 4-(4-methoxy-phenylamino)-3-phenoxy-3, 4-dihydro-1 $H$ quinolin-2-one (7a) as a white solid, which was recrystallized from ethyl acetate-pet-ether 
mixture. Yield, $0.162 \mathrm{~g}, 90 \%$; white solid, mp $229{ }^{\circ} \mathrm{C}$; IR $\left(\mathrm{CHCl}_{3}\right) 3373,1686 \mathrm{~cm}^{-1}$; ${ }^{1} \mathrm{H}$ NMR $\left(\mathrm{CDCl}_{3}+\mathrm{DMSO}_{-} \mathrm{d}_{6}, 200 \mathrm{MHz}\right) \delta 10.42(\mathrm{~s}, 1 \mathrm{H}), 7.69(\mathrm{~m}, 1 \mathrm{H}), 7.49(\mathrm{~m}, 3 \mathrm{H}), 7.24(\mathrm{~m}, 5 \mathrm{H}), 7.25(\mathrm{~d}$, $J=9.0 \mathrm{~Hz}, 2 \mathrm{H}), 6.93(\mathrm{~d}, J=9.0 \mathrm{~Hz}, 2 \mathrm{H}), 5.23(\mathrm{~d}, J=8.6 \mathrm{~Hz}, 1 \mathrm{H}), 5.13(\mathrm{~d}, J=8.6 \mathrm{~Hz}, 1 \mathrm{H}), 4.01$ $(\mathrm{s}, 3 \mathrm{H}) ;{ }^{13} \mathrm{C}$ NMR $\left(\mathrm{DMSO}-\mathrm{d}_{6}, 125.76 \mathrm{MHz}\right) \delta 167.11,158.05,152.16,135.53,128.76,128.48$, $127.49,122.61,121.31,115.84,115.51,114.99,114.24,75.86,55.97,55.16$. Anal. Calcd. for $\mathrm{C}_{22} \mathrm{H}_{20} \mathrm{~N}_{2} \mathrm{O}_{3}$ : C, 73.30; H, 5.60; N, 7.77. Found: C, 73.00; H, 5.43; N, 7.65.

Transfer hydrogenation of 1-(4-methoxyphenyl)-4-(2-nitrophenyl)-3-phenoxyazetidin-2-one (5a) in refluxing methanol. To a solution of $5 a(0.195 \mathrm{~g}, 0.5 \mathrm{mmol})$ in dry methanol $(3 \mathrm{~mL})$ was added ammonium formate $(0.157 \mathrm{~g}, 2.5 \mathrm{mmol})$ followed by $\mathrm{Pd} / \mathrm{C}(10 \%, 30 \mathrm{mg})$. This reaction mixture was refluxed with stirring for $2 \mathrm{~h}$ till the starting material was consumed completely (TLC). The reaction mixture was cooled to room temperature, filtered through a short celite bed and the bed was washed with methylene chloride $(15 \mathrm{~mL})$. The filtrate was diluted with water $(2 \mathrm{~mL})$, the organic layer was separated and dried over anhyd. $\mathrm{Na}_{2} \mathrm{SO}_{4}$. Solvent was removed under reduced pressure to get white solid $(0.172 \mathrm{~g})$. It was found to be a 2:1 mixture of 4-(2aminophenyl)-1-p-methoxyphenyl-3-phenoxy azetidin-2-one (6), 4-(4-methoxy-phenylamino)-3phenoxy-3, 4-dihydro-1H-quinolin-2-one (7a) along with an unidentified product. The compounds 6 and 7a (total $0.13 \mathrm{~g}, 70 \%$ ) was obtained as a mixture by column chromatography.

\section{General procedure for microwave assisted synthesis of 3,4-dihydro-1H-quinolin-2-one (7a-k)}

To a solution of azetidin-2-one $(5,0.5 \mathrm{mmol})$, in ethylene glycol $(3 \mathrm{~mL})$ was added ammonium formate $(2.5 \mathrm{mmol})$ followed by $\mathrm{Pd} / \mathrm{C}(10 \%, 30 \mathrm{mg})$. The mixture was then subjected to microwave irradiation at low power setting $(60 \%)$ for $3 \mathrm{~min}$. in an open glass vessel. It was then allowed to come to room temperature, diluted with water $(2 \mathrm{~mL})$ and filtered through a small pad of celite. The residue was washed with methylene chloride $(2 \times 10 \mathrm{~mL})$, the organic layer was separated, washed with brine $(2 \mathrm{~mL})$, dried over anhyd. $\mathrm{Na}_{2} \mathrm{SO}_{4}$ and the solvent was removed in vacuo to get crude quinolinone, which was recrystallized from an ethyl acetate-pet-ether mixture. 4-(4-Methoxy-phenylamino)-3-phenoxy-3,4-dihydro-1H-quinolin-2-one (7a). Yield, $0.160 \mathrm{~g}$, $89 \%$; physical and spectral data was same as obtained earlier.

3-Methoxy-4-(4-Methoxy-phenylamino)-3,4-dihydro-1H-quinolin-2-one (7b). Yield, $0.134 \mathrm{~g}$, 90\%; white solid, mp $208{ }^{\circ} \mathrm{C}$; IR $\left(\mathrm{CHCl}_{3}\right) 3387,1678 \mathrm{~cm}^{-1} ;{ }^{1} \mathrm{H}$ NMR $\left(\mathrm{CDCl}_{3}+\mathrm{DMSO}-\mathrm{d}_{6}, 500\right.$ MHz) $\delta 9.69(\mathrm{~s}, 1 \mathrm{H}), 7.06-6.94(\mathrm{~m}, 2 \mathrm{H}), 6.73-6.69(\mathrm{~m}, 2 \mathrm{H}), 6.48(\mathrm{~d}, J=9.2 \mathrm{~Hz}, 2 \mathrm{H}), 6.39(\mathrm{~d}, J=$ $8.7 \mathrm{~Hz}, 2 \mathrm{H}), 4.31(\mathrm{~d}, J=7.8 \mathrm{~Hz}, 1 \mathrm{H}), 3.74(\mathrm{~d}, J=7.8 \mathrm{~Hz}, 1 \mathrm{H}), 3.47(\mathrm{~s}, 3 \mathrm{H}), 3.26(\mathrm{~s}, 3 \mathrm{H}) ;{ }^{13} \mathrm{C}$ NMR $\left(\mathrm{DMSO}_{-} \mathrm{d}_{6}, 125.76 \mathrm{MHz}\right) \delta 168.06,151.76,140.28,135.38,128.16,128.09,123.49$, 122.29, 115.25, 114.39, 114.14, 77.52, 58.12, 55.30, 54.99; Anal. Calcd. for $\mathrm{C}_{17} \mathrm{H}_{18} \mathrm{~N}_{2} \mathrm{O}_{3}$ : C, 68.43; H, 6.09, N, 9.39. Found: C, 68.72; H, 6.20; N, 9.11.

3-Benzyloxy-4-(4-Methoxy-phenylamino)-3,4-dihydro-1H-quinolin-2-one $\quad$ (7c). Yield, $0.159 \mathrm{~g}, 85 \%$; white solid, mp $201{ }^{\circ} \mathrm{C}$; IR $\left(\mathrm{cm}^{-1}\right) 3367,1680 ;{ }^{1} \mathrm{H} \mathrm{NMR}\left(\mathrm{CDCl}_{3}, 500 \mathrm{MHz}\right) \delta 8.41$ (bs, 1H), 7.12-7.06 (m, 5H), 7.04-6.99 (m, 2H), 6.82-6.78 (m, 1H), 6.68-6.63 (m, 1H), $6.48(\mathrm{~d}$, $J=8.8 \mathrm{~Hz}, 2 \mathrm{H}), 6.27-6.22(\mathrm{~m}, 2 \mathrm{H}), 4.97(\mathrm{~d}, J=11.5 \mathrm{~Hz}, 1 \mathrm{H}), 4.41$ (d, $J=12.1 \mathrm{~Hz}, 1 \mathrm{H}), 4.35$ (d, 
$J=7.7 \mathrm{~Hz}, 1 \mathrm{H}), 4.03(\mathrm{bs}, 1 \mathrm{H}), 3.50(\mathrm{~s}, 3 \mathrm{H}) ;{ }^{13} \mathrm{C}$ NMR $\left(\mathrm{DMSO}_{-} \mathrm{d}_{6}, 75.48 \mathrm{MHz}\right) \delta 168.31,151.46$, 141.82 , 138.10, 136.87, 128.91, 128.70, 128.36, 128.15, 127.78, 124.33, 122.59, 115.66, 114.90, 114.14, 76.02, 72.08, 55.57, 55.23; Anal. Calcd. for $\mathrm{C}_{23} \mathrm{H}_{22} \mathrm{~N}_{2} \mathrm{O}_{3}$ : C, 73.77; H, 5.93; N, 7.48. Found: C, 73.53; H, 5.84; N, 7.32.

3-Phenoxy-4-phenylamino-3,4-dihydro-1H-quinolin-2-one (7d). Yield, 0.144 g, 87\%; fluppy white solid, mp $225{ }^{\circ} \mathrm{C}$; IR $\left(\mathrm{CHCl}_{3}\right) 3383,1682 \mathrm{~cm}^{-1} ;{ }^{1} \mathrm{H}$ NMR $\left(\mathrm{CDCl}_{3}+\mathrm{DMSO}_{6}, 500 \mathrm{MHz}\right) \delta$ $9.73(\mathrm{~s}, 1 \mathrm{H}), 6.69(\mathrm{~d}, J=7.3 \mathrm{~Hz}, 1 \mathrm{H}), 6.56-6.49(\mathrm{~m}, 3 \mathrm{H}), 6.42-6.39(\mathrm{~m}, 2 \mathrm{H}), 6.32-6.30(\mathrm{~m}, 2 \mathrm{H})$, 6.24-6.22 (m, 3H), $6.01(\mathrm{~d}, J=7.8 \mathrm{~Hz}, 2 \mathrm{H}), 5.94-5.91(\mathrm{~m}, 1 \mathrm{H}), 4.29(\mathrm{~d}, J=9.1 \mathrm{~Hz}, 1 \mathrm{H}), 4.25(\mathrm{~d}$, $J=9.2 \mathrm{~Hz}, 1 \mathrm{H}) ;{ }^{13} \mathrm{C}$ NMR $\left(\mathrm{DMSO}_{6}, \mathrm{~d}_{6}, 125.76 \mathrm{MHz}\right) \delta 165.98,157.27,146.33,134.85,127.60$, 127.49, 127.14, 126.16, 122.77, 121.20, 119.95, 115.34, 114.81, 114.27, 111.45, 74.95, 53.01; Anal. Calcd. for $\mathrm{C}_{21} \mathrm{H}_{18} \mathrm{~N}_{2} \mathrm{O}_{2}$ : C, 76.33; H, 5.50, N, 8.48. Found: C, 76.51; H, 5.58; N, 8.19.

3-Methoxy-4-phenylamino-3,4-dihydro-1H-quinolin-2-one (7e). Yield, $0.108 \mathrm{~g}$, 81\%; white crystalline solid, mp $209^{\circ} \mathrm{C}$; IR $\left(\mathrm{CHCl}_{3}\right) 3392,1687 \mathrm{~cm}^{-1} ;{ }^{1} \mathrm{H} \mathrm{NMR}\left(\mathrm{CDCl}_{3}, 500 \mathrm{MHz}\right) \delta 9.08(\mathrm{~s}$, $1 \mathrm{H}), 7.35(\mathrm{~d}, J=7.4 \mathrm{~Hz}, 1 \mathrm{H}), 7.14(\mathrm{~m}, 3 \mathrm{H}), 6.96(\mathrm{~m}, 1 \mathrm{H}), 6.84(\mathrm{~d}, J=7.8 \mathrm{~Hz}, 1 \mathrm{H}), 6.72(\mathrm{~m}, 1 \mathrm{H})$, $6.62(\mathrm{~d}, J=7.8 \mathrm{~Hz}, 2 \mathrm{H}), 4.64(\mathrm{~d}, J=7.3 \mathrm{~Hz}, 1 \mathrm{H}), 4.06(\mathrm{~d}, J=7.3 \mathrm{~Hz}, 1 \mathrm{H}), 3.49(\mathrm{~s}, 1 \mathrm{H}) ;{ }^{13} \mathrm{C}$ NMR $\left(\mathrm{CDCl}_{3}, 125.76 \mathrm{MHz}\right) \delta 169.20,146.16,135.17,129.38,129.25,128.82,123.83,123.57$, 118.87, 116.01, 113.97, 77.90, 58.90, 55.37; Anal. Calcd. for $\mathrm{C}_{16} \mathrm{H}_{16} \mathrm{~N}_{2} \mathrm{O}_{2}$ : C, 71.61; $\mathrm{H}, 6.02 ; \mathrm{N}$, 10.44. Found: C, 71.32; H, 6.00; N, 10.15 .

3-Benzyloxy-4-phenylamino-3,4-dihydro-1H-quinolin-2-one (7f). Yield, $0.141 \mathrm{~g}$, 82\%; white solid, mp $218{ }^{\circ} \mathrm{C}$; IR $\left(\mathrm{CHCl}_{3}\right) 3389,1680 \mathrm{~cm}^{-1} ;{ }^{1} \mathrm{H} \mathrm{NMR}\left(\mathrm{CDCl}_{3}, 200 \mathrm{MHz}\right) \delta 8.79(\mathrm{~s}, 1 \mathrm{H}), 7.34$ $(\mathrm{m}, 6 \mathrm{H}), 7.12(\mathrm{~m}, 3 \mathrm{H}), 6.92(\mathrm{~d}, J=7.9 \mathrm{~Hz}, 1 \mathrm{H}), 6.78(\mathrm{~m}, 1 \mathrm{H}), 6.53(\mathrm{~d}, J=7.8 \mathrm{~Hz}, 2 \mathrm{H}), 4.97(\mathrm{~d}$, $J=11.7 \mathrm{~Hz}, 1 \mathrm{H}), 4.70(\mathrm{~m}, 2 \mathrm{H}), 4.28(\mathrm{~d}, J=7.4 \mathrm{~Hz}, 1 \mathrm{H}) ;{ }^{13} \mathrm{C}$ NMR (DMSO-d $\left.6,75.48 \mathrm{MHz}\right) \delta$ $168.22,147.80,138.10,136.94,129.15,128.88,128.82,128.42,128.18,127.87,124.03,122.68$, 116.61, 115.69, 112.76, 75.84, 72.05, 54.26. Anal. Calcd. for $\mathrm{C}_{22} \mathrm{H}_{20} \mathrm{~N}_{2} \mathrm{O}_{2}: \mathrm{C}, 76.71 ; \mathrm{H}, 5.86 ; \mathrm{N}$, 8.13. Found: C, 76.51; H, 6.00; N, 7.90.

3-Acetoxy-4-phenylamino-3,4-dihydro-1H-quinolin-2-one (7g). Yield, 0.109 g, 74\%; fluppy white solid, mp $221{ }^{\circ} \mathrm{C}$; IR $\left(\mathrm{CHCl}_{3}\right) 3381,1757,1691 \mathrm{~cm}^{-1} ;{ }^{1} \mathrm{H}$ NMR $\left(\mathrm{CDCl}_{3}+\mathrm{DMSO}-\mathrm{d}_{6}, 200\right.$ $\mathrm{MHz}) \delta 10.24(\mathrm{~s}, 1 \mathrm{H}), 7.35(\mathrm{~d}, J=7.4 \mathrm{~Hz}, 1 \mathrm{H}), 7.13(\mathrm{~m}, 4 \mathrm{H}), 6.93(\mathrm{~m}, 2 \mathrm{H}), 6.68(\mathrm{~d}, J=7.9 \mathrm{~Hz}$, 2H), $5.51(\mathrm{~d}, J=11.7 \mathrm{~Hz}, 1 \mathrm{H}), 4.93(\mathrm{~d}, J=11.0 \mathrm{~Hz}, 1 \mathrm{H}), 1.89$ (s, 1H); ${ }^{13} \mathrm{C}$ NMR (DMSO-d 6 , $125.76 \mathrm{MHz}) \delta 169.75,165.77,146.81,135.18,128.50,128.28,126.04,123.51,122.51,117.10$, 115.36, 112.73, 70.94, 53.36, 19.87. Anal. Calcd. for $\mathrm{C}_{17} \mathrm{H}_{16} \mathrm{~N}_{2} \mathrm{O}_{3}$ : C, 68.89; H, 5.45; N, 9.45; Found: C, 68.71; H, 5.20; N, 9.12.

3-Phenoxy-4-p-tolylamino-3,4-dihydro-1H-quinolone-2-one (7h). Yield, $0.150 \mathrm{~g}$, 87\%; fluppy white solid, mp $248{ }^{\circ} \mathrm{C}$; IR $\left(\mathrm{CHCl}_{3}\right) 3360,1690 \mathrm{~cm}^{-1} ;{ }^{1} \mathrm{H} \mathrm{NMR}\left(\mathrm{CDCl}_{3}+\mathrm{DMSO}-\mathrm{d}_{6}, 500 \mathrm{MHz}\right) \delta$ $9.73(\mathrm{~s}, 1 \mathrm{H}), 6.69(\mathrm{~d}, J=7.3 \mathrm{~Hz}, 1 \mathrm{H}), 6.56-6.51(\mathrm{~m}, 3 \mathrm{H}), 6.33-6.30(\mathrm{~m}, 2 \mathrm{H}), 6.26-6.23(\mathrm{~m}, 5 \mathrm{H})$, $5.94(\mathrm{~d}, J=8.3 \mathrm{~Hz}, 2 \mathrm{H}), 4.25(\mathrm{~s}, 2 \mathrm{H}), 1.53(\mathrm{~s}, 3 \mathrm{H}) ;{ }^{13} \mathrm{C}$ NMR (DMSO-d $\left.6,125.76 \mathrm{MHz}\right) \delta$ $165.97,157.27,143.82,134.86,128.01,127.64,127.14,126.32,124.22,122.85,121.22,119.98$, 114.84, 114.29, 111.72, 74.86, 53.42, 18.77; Anal. Calcd. for $\mathrm{C}_{22} \mathrm{H}_{20} \mathrm{~N}_{2} \mathrm{O}_{2}$ : C, 76.71; $\mathrm{H}, 5.86$; N, 8.14. Found: C, 76.53; H, 5.68; N, 7.92. 
3-Methoxy-4-p-tolylamino-3,4-dihydro-1-H-quinolin-2-one (7i). Yield, $0.121 \mathrm{~g}$, 86\%; white crystalline solid, mp $211^{\circ} \mathrm{C}$; IR $\left(\mathrm{CHCl}_{3}\right) 3373,1689 \mathrm{~cm}^{-1} ;{ }^{1} \mathrm{H} \mathrm{NMR}\left(\mathrm{CDCl}_{3}, 200 \mathrm{MHz}\right) \delta 8.85(\mathrm{~s}$, $1 \mathrm{H}), 7.36-7.23(\mathrm{~m}, 2 \mathrm{H}), 7.08-6.89(\mathrm{~m}, 4 \mathrm{H}), 6.63(\mathrm{~d}, J=6.7 \mathrm{~Hz}, 2 \mathrm{H}), 4.69$ (d, $J=7.4 \mathrm{~Hz}, 1 \mathrm{H})$, $4.14(\mathrm{~d}, J=7.4 \mathrm{~Hz}, 1 \mathrm{H}), 3.58(\mathrm{~s}, 3 \mathrm{H}), 2.27(\mathrm{~s}, 3 \mathrm{H}) ;{ }^{13} \mathrm{C}$ NMR $\left(\mathrm{DMSO}-\mathrm{d}_{6}, 75.48 \mathrm{MHz}\right) \delta 167.74$, $145.31,136.58,129.38,128.50,128.34,124.83,124.13,122.24,115.34,112.72,78.05,57.93$, 53.94, 19.97; Anal. Calcd. for $\mathrm{C}_{17} \mathrm{H}_{18} \mathrm{~N}_{2} \mathrm{O}_{2}$ : C, 72.31; H, 6.44; N, 9.92. Found: C, 72.10; H, 6.53; N, 9.83 .

3-Benzyloxy-4-p-tolylamino-3,4-dihydro-1-H-quinolin-2-one (7j). Yield, 0.139 g, 78\%; fluppy white solid, mp $203{ }^{\circ} \mathrm{C}$; IR $\left(\mathrm{CHCl}_{3}\right) 3371,1677 \mathrm{~cm}^{-1} ;{ }^{1} \mathrm{H}$ NMR $\left(\mathrm{CDCl}_{3}+\mathrm{DMSO}-\mathrm{d}_{6}, 500\right.$ $\mathrm{MHz}) \delta 8.46(\mathrm{bs}, 1 \mathrm{H}), 7.25(\mathrm{~m}, 5 \mathrm{H}), 7.20-7.17(\mathrm{~m}, 2 \mathrm{H}), 6.95-6.80(\mathrm{~m}, 2 \mathrm{H}), 6.39(\mathrm{~m}, 2 \mathrm{H}), 4.83$ $(\mathrm{d}, J=11.9 \mathrm{~Hz}, 1 \mathrm{H}), 4.58(\mathrm{~d}, J=12.0 \mathrm{~Hz}, 1 \mathrm{H}), 4.54(\mathrm{~d}, J=7.4 \mathrm{~Hz}, 1 \mathrm{H}) 4.24(\mathrm{bs}, 1 \mathrm{H}), 2.16$ (s, $3 \mathrm{H}) ;{ }^{13} \mathrm{C}$ NMR (DMSO-d $\left.6,75.48 \mathrm{MHz}\right) \delta 168.11,145.31,137.93,136.74,129.41,128.68$, $128.56,128.19,127.95,127.64,124.92,124.07,122.45,115.49,112.81,75.76,71.88,54.45$, 20.06; Anal. Calcd. for $\mathrm{C}_{23} \mathrm{H}_{22} \mathrm{~N}_{2} \mathrm{O}_{2}$ : C, 77.06; H, 6.20; N, 7.82. Found: C, 76.81; H, 6.10; N, 7.56 .

3-Acetoxy-4-p-tolylamino-3,4-dihydro-1-H-quinolin-2-one (7k). Yield, 0.119 g, 77\%; fluppy white solid, mp $238{ }^{\circ} \mathrm{C}$; IR $\left(\mathrm{CHCl}_{3}\right) 3379,1755,1693 \mathrm{~cm}^{-1} ;{ }^{1} \mathrm{H}$ NMR $\left(\mathrm{CDCl}_{3}+\mathrm{DMSO}-\mathrm{d}_{6}, 500\right.$ $\mathrm{MHz}) \delta 9.94(\mathrm{~s}, 1 \mathrm{H}), 6.97(\mathrm{~d}, J=7.3 \mathrm{~Hz}, 1 \mathrm{H}), 6.80-6.77(\mathrm{~m}, 2 \mathrm{H}), 6.58-6.51(\mathrm{~m}, 4 \mathrm{H}), 6.22(\mathrm{~d}, J=$ $8.3 \mathrm{~Hz}, 2 \mathrm{H}), 5.09(\mathrm{~d}, J=12.5 \mathrm{~Hz}, 1 \mathrm{H}), 4.50(\mathrm{~d}, J=11.5 \mathrm{~Hz}, 1 \mathrm{H}), 1.79(\mathrm{~s}, 3 \mathrm{H}), 1.54(\mathrm{~s}, 3 \mathrm{H}) ;{ }^{13} \mathrm{C}$ NMR (DMSO-d $6,125.76 \mathrm{MHz}) \delta 169.16,165.39,144.31,134.92,128.59,127.78,125.58$, $125.41,123.45,122.01,114.91,112.46,70.54,53.13,19.58,19.31$; Anal. Calcd. for $\mathrm{C}_{18} \mathrm{H}_{18} \mathrm{~N}_{2} \mathrm{O}_{3}: \mathrm{C}, 69.65 ; \mathrm{H}, 5.86 ; \mathrm{N}, 9.03$. Found: $\mathrm{C}, 69.53 ; \mathrm{H}, 5.67 ; \mathrm{N}, 9.00$.

\section{Acknowledgements}

The authors thank the Department of Science and Technology, New Delhi for financial support and NMS thanks UGC, New Delhi for a research fellowship.

\section{References}

1. For reviews on $\beta$-lactam antibiotics, see: (a) Dürkheimer, W.; Blumbach, J.; Lattrell, R.; Scheunemann, K. H. Angew. Chem., Int. Ed. 1985, 24, 180. (b) Chemistry and Biology of $\beta$ Lactam Antibiotics, Morin, R. B.; Gorman, M., Eds; Academic: New York, 1982; Vol. 1-3. (c) Coulton, S.; Hunt, E. In Recent Progress in the Chemical Synthesis of Antibiotics and Related Microbial Products; Lukacs, G., Ed.; Springer-Verlag: Berlin, 1993; Vol. 2, p 621. (d) Southgate, R. Contemp. Org. Synth. 1994, 1, 417.

2. The Chemistry of $\beta$-lactams, Page, M. I.; Ed.; Chapman and Hall: London, 1992. 
3. For comprehensive general reviews, see: (a) Koppel, G. A. In Small Ring Heterocycles; Hassner, A., Ed.; Wiley: New York, 1983; Vol. 42, p 219. (b) Backes, J. In Houben-Weyl, Methoden der Organischem Chemie; Muller, E.; Bayer, O., Eds; Thieme: Stuttgart, 1991; Band E16B, p 31. (c) De Kimpe, N. In Comprehensive Heterocyclic Chemistry II, Katritzky, A. R.; Rees, C. W.; Scriven, E. F.V.; Padwa, A., Eds; Pergamon: Oxford, 1996, Vol.1B, p 507.

4. (a) Ojima, I. In The Chemistry of $\beta$-Lactams Georg, G. I. Ed.; VCH: New York, 1993; p 197.

(b) Palomo, C.; Aizpurua, J.; Ganboa, I. In Enantioselective Synthesis of Beta-Amino Acids Juaristi, E. Ed. Wiley-VCH: New York, 1997; p 279 and references cited therein. (c) For a review on this subject see: Ojima, I.; Delaloge, F. Chem. Soc. Rev. 1997, 26, 377. (d) Alcaide, B.; Almendros, P. Chem. Soc. Rev. 2001, 30, 226. (e) Alcaide, B.; Almendros, P. Synlett 2002, 381.

5. Alcaide, B.; Almendros, P. Current Medicinal Chemistry 2004, 11, 1921.

6. Adlington, R. M.; Baldwin, J. E.; Chen, B.; Cooper, S. L.; McCoull, W.; Pritchard, G. J.; Howe, T. J.; Becker, G. W.; Hermann, R. B.; McNulty, A. M.; Neubauer, B. L. Bioorg. Med. Chem. Lett. 1997, 7, 1689.

7. Han, W. T.; Trehan, A. K.; Kim Wright, J. J.; Federeci, M. E.; Seiler, S. M.; Meanwell, N. A. Bioorg. Med. Chem. 1995, 3, 1123.

8. Borthwick, A. D.; Weingarten, G.; Haley, T. M.; Tomaszewski, M.; Wang, W.; Hu, Z.; Bedard, J.; Jih, H.; Yuen, L.; Mansour, T. S. Bioorg. Med. Chem. Lett. 1998, 8, 365.

9. (a) Burnett, D. A.; Caplen, M. A.; Davis, H. R., Jr.; Burrier, R. E.; Clader, J. W. J. Med. Chem. 1994, 37, 1733. (b) Dugar, S.; Yumibe, N.; Clader, J. W.; Vizziano, M.; Huie, K.; van Heek, M.; Compton, D. S.; Davis, H. R., Jr. Bioorg. Med. Chem. Lett. 1996, 6, 1271. (c) Wu, G. G. Org. Process Res. Dev. 2000, 4, 298.

10. (a) Doherty, J. B.; Ashe, B. M.; Agrenbright, L. W.; Barker, P. L.; Bonney, R. J.; Chandler, G. O.; Dahlgren, M. E.; Dorn, C. P., Jr.; Finke, P. E.; Firestone, R. A.; Fletcher, D.; Hagemann, W. K.; Munford, R.; O’Grady, L.; Maycock, A. L.; Pisano, J. M.; Shah, S. K.; Thomson, K. R.; Zimmerman, M. Nature 1986, 322, 192. (b) Cvetovich, R. J.; Chartran, M.; Hartner, F. W.; Roberge, C.; Amato, J. S.; Grabowski, E. J. J. Org. Chem. 1996, 61, 6575.

11. (a) Zhou, N. E.; Guo, D.; Thomas, G.; Reddy, A. V. N.; Kaleta, J.; Purisima, E.; Menard, R.; Micetich, R. G.; Singh, R. Bioorg. Med. Chem. Lett. 2003, 13, 139. (b) Setti, E. L.; Davis, D.; Chung, T.; McCarter, J. Bioorg. Med. Chem. Lett. 2003, 13, 2051.

12. Staudinger, H. Liebigs Ann. Chem. 1907, 356, 51.

13. (a) Palomo, C.; Aizpurua, J.; Mielgo, A.; Linden, A. J. Org. Chem. 1996, 61, 9186. (b) Palomo, C.; Aizpurua, J. M.; Ganboa, I.; Oirabide, M. Eur. J. Org. Chem. 1999, 8, 3223. (c) Alcaide, B.; Almendros, P. Chem. Soc. Rev. 2001, 30, 226.

14. (a) Govande, V. V.; Arun, M.; Deshmukh, A. R. A. S.; Bhawal, B. M. Synth. Commun. 2000, 30, 4177. (b) Joshi, S. N.; Deshmukh, A. R. A. S.; Bhawal, B. M. Tetrahedron: Asymmetry 2000, 11, 1477. (c) Krishnaswamy, D.; Bhawal, B. M.; Deshmukh, A. R. A. S. Tetrahedron Lett. 2000, 41, 417. (d) Thiagarajan, K.; Puranik, V. G.; Deshmukh, A. R. A. S.; Bhawal, B. 
M. Tetrahedron 2000, 56, 7811. (e) Karupaiyan, K.; Puranik, V. G.; Deshmukh, A. R. A. S.; Bhawal, B. M. Tetrahedron 2000, 56, 8555. (f) Bhawal, B. M.; Joshi, S. N.; Krishnaswamy, D.; Deshmukh, A. R. A. S. J. Indian Inst. Sci. 2001, 81, 265. (g) Joshi, S. N.; Puranik, V. G.; Deshmukh, A. R. A. S.; Bhawal, B. M. Tetrahedron: Asymmetry 2001, 12, 3073. (h) Arun, M.; Govande, V. V.; Deshmukh, A. R. A. S. Bhawal, B. M. Indian J. Chem. 2002, 41B, 856. (i) Krishnaswamy, D.; Govande, V. V.; Gumaste, V. K.; Bhawal, B. M.; Deshmukh, A. R. A. S. Tetrahedron 2002, 58, 2215. (j) Patil, R. T.; Parveen, G.; Gumaste, V. K.; Bhawal, B. M.; Deshmukh, A. R. A. S. Synlett 2002, 1455. (k) Joshi, S. N.; Phalgune, U. D.; Bhawal, B. M.; Deshmukh, A. R. A. S. Tetrahedron Lett. 2003, 44, 1827. (1) Arun, M.; Joshi, S. N.; Puranik, V. G.; Bhawal, B. M.; Deshmukh, A. R. A. S. Tetrahedron 2003, 59, 2309. (m) Shinkre, B. A.; Puranik, V. G.; Bhawal, B. M.; Deshmukh A. R. A. S. Tetrahedron: Asymmetry 2003, 14, 453. (n) Jayanthi, A.; Gumaste, V. K.; Deshmukh, A. R. A. S. Synlett 2004, 979.

15. (a) Jayaraman, M.; Deshmukh, A. R. A. S.; Bhawal, B. M. Tetrahedron 1996, 52, 8989. (b) Jayaraman, M.; Puranik, V. G.; Bhawal, B. M. Tetrahedron 1996, 52, 9005. (c) Srirajan, V.; Deshmukh, A. R. A. S.; Puranik, V. G.; Bhawal, B. M. Tetrahedron: Asymmetry 1996, 7, 2733. (d) Krishnaswamy, D.; Govande, V. V.; Deshmukh, A. R. A. S. Synthesis 2003, 12, 1903. (e) Deshmukh, A. R. A. S.; Bhawal, B. M.; Krishnaswamy, D.; Govande, V. V.; Shinkre, B. A.; Jayanthi, A. Current Medicinal Chemistry 2004, 11, 1889.

16. Pei, Y.; Houghten, R. A.; Kiely, J. S. Tetrahedron Lett. 1997, 38, 3349.

17. (a) Hayes, B. L. Microwave Synthesis: Chemistry at Speed of Light, CEM Publishing: Mathews, NC, USA, 2002. (b) Varma, R. S. Microwaves in Organic Syntheses, Loupy, A., Ed.; Wiley-VCH: New York, 2002; pp181-218. (c) Varma, R. S. Advances in Green Chemistry: Chemical Syntheses using Microwave Irradiation, Astra Zeneca, Research Foundation: Banglore, India, 2003. (d) Deshayes, S.; Liagre, M.; Loupy, A.; Luche, J. L.; Petit, A. Tetrahedron 1999, 55, 10851. (e) Bose, A. K.; Banik, B. K.; Lavlinskaia, N.; Jayaraman, M.; Manhas, M. S. Chemtech 1997, 18. (f) Caddick, S. Tetrahedron 1995, 51, 10403. 